\title{
Quality of life of people living with HIV and AIDS and antiretroviral therapy
}

\author{
This article was published in the following Dove Press journal: \\ HIVIAIDS - Research and Palliative Care \\ 6 August 2012 \\ Number of times this article has been viewed
}

\section{Oluwafemi O Oguntibeju \\ Oxidative Stress Research Centre, Cape Peninsula University of Technology, Bellville, South Africa}

\begin{abstract}
The development of antiretroviral drugs has significantly changed the perception of HIV/AIDS from a very fatal to a chronic and potentially manageable disease, and the availability and administration of antiretroviral therapy (ART) has significantly reduced mortality and morbidity associated with HIV and AIDS. There is a relationship between ART and quality of life of people living with HIV and AIDS, and several studies have reported a strong positive association between ART and improved quality of life in different domains among people living with HIV and AIDS in both developed and developing countries. However, a few studies have reported on the negative effects of ART, which directly or indirectly relate to the quality of life and longevity of HIV-infected persons. In this review, the effects and benefits of ART on people living with HIV and AIDS based on studies done in developed and developing countries is examined.
\end{abstract}

Keywords: benefits, negative effects, oxidative stress, treatment, modifications of desires

\section{Introduction}

In July 2008, the Joint United Nations Programme on HIV/AIDS (UNAIDS) reported that by December 2007, there were an estimated 33.0 million people globally living with HIV infection. It also stated that the number of cases of new HIV infections had decreased from 3.0 million in 2001 to 2.7 million in 2007. The report showed that 2.0 million people died globally due to AIDS-related disease in 2007, compared with an estimated 1.7 million AIDS-related deaths in 2001. The report further stated that although the percentage of people who are living with HIV appeared to have stabilized, the overall number of people living with HIV infection had steadily increased, as new HIV infections occur on a yearly basis. According to the UNAIDS report, Southern Africa continues to bear a disproportionate burden of HIV infection, with $35 \%$ of HIV infection and 38\% of AIDS-related deaths reported in Southern Africa in 2007. In total, sub-Saharan Africa is reported to be home to $67 \%$ of all people living with HIV. ${ }^{1}$ As the HIV/AIDS pandemic continues to expand, especially in developing countries, the moral obligation to provide safe and efficient antiretroviral treatment has become apparent to both national and international health-care communities. Standard antiretroviral therapy (ART) consists of the use of at least three antiretroviral (ARV) drugs to suppress maximally the HIV and stop the progression of HIV disease. There have been significant reductions seen in the rates of death and suffering following the use of a potent ARV regimen. The World Health Organization (WHO) and UNAIDS estimate that at least 14.6 million people were in need of antiretroviral therapy in 2009. 
As of the end of 2009, 5.25 million people had access to antiretroviral therapy in low- and middle-income countries.

\section{Antiretroviral therapy: general background}

Access to ARV drugs is increasing among people living with HIV and AIDS in developing countries due to local, national, and international efforts. ${ }^{2}$ Potent combination ART, mainly consisting of three or more ARV drugs, has greatly improved the health and survival rates of HIV-infected patients in areas of the world with access to ARVs. More than 20 individual ARVs in six classes are available in the United States, in addition to several fixed-dose combination preparations. These can be combined to construct a number of effective regimens for initial and subsequent therapy. Although ART has its limitations, it saves lives and improves immune system function, reduces the risk of many HIV-related and "non-AIDS" complications, and reduces the risk of HIV transmission. Increasingly, several lines of evidence point to the benefit of ART even for patients with high CD4 counts. ${ }^{3}$

The mortality and morbidity benefits of ART are obvious in patients with relatively advanced immune suppression or with symptoms related to HIV infection. For asymptomatic patients with higher CD4 counts (eg, $>350$ cells $/ \mathrm{mm}^{3}$ ), the question of when to initiate ART remains an area of research and debate. However, it is known that there is a spectrum of risk for adverse outcomes that increases as the CD4 count declines. In persons with CD4 counts of $<200$ cells $/ \mu \mathrm{L}$, effective ART dramatically decreases morbidity and mortality. For persons with CD4 counts of 200-350 cells $/ \mathrm{mm}^{3}$, data from randomized controlled studies as well as cohort studies also demonstrate a reduction in both AIDS and non-AIDS events among those who start ART. A variety of data from observational cohort studies show a reduction in death as well as in AIDS and non-AIDS related complications among persons who start ART with CD4 counts of $>350$ cells $/ \mu \mathrm{L}$ rather than $<350$ cells $/ \mathrm{mm}^{3} .4,5$

\section{Classes of ART and mechanisms of action}

ARVs have been shown to reduce significantly the rate of replication of HIV in the body of an HIV-infected person. Although ART does not completely destroy the virus and cannot cure the infection/disease, it can however greatly decrease the viral load and significantly slow the progression of the disease, thereby increasing life expectancy and improving the quality of life of people living with HIV and AIDS. ART is not required by all people living with HIV and AIDS at all stages of HIV infection, but ART is recommended when the $\mathrm{CD} 4$ is less than 350 cells $/ \mathrm{mm}^{3}$. ART provides effective treatment options for treatment-naive and treatment-experienced patients. Six classes of antiretroviral agents currently exist: nucleoside/nucleotide reverse-transcriptase inhibitors (NRTIs), nonnucleoside reverse transcriptase inhibitors (NNRTIs), protease inhibitors (PIs), integrase inhibitors, fusion inhibitors, and chemokine receptor antagonists. Each class of ART targets a different step in the viral life cycle as the virus infects a $\mathrm{CD} 4^{+} \mathrm{T}$ lymphocyte or other target cells. The use of these agents in clinical practice is dictated by their ease or complexity of use, effect profile, efficacy based on clinical evidence, practice guidelines, and clinician preference. ${ }^{6}$ The NRTIs were the first agents available for the treatment of HIV infection. Although NRTIs have been found to be less potent against HIV than NNRTIs and PIs, the NRTIs have had a principal role in ARV treatment, remain part of the current standard of care, and have been shown to exhibit activity against HIV-1 and HIV-2., $3,6,7$

When HIV infects a cell, the viral RNA is converted into viral DNA and is then copied into the host cell's DNA by an enzyme called reverse transcriptase. The viral DNA then instructs the cell to make copies of HIV genetic material. The protease enzyme assembles the copied viral genetic material into new viruses, after which they are released from the cell to infect other cells. ${ }^{3,6}$

The first class of ARV reverse-transcriptase inhibitors operates early in the HIV life cycle to stop viral replication following HIV infection. There are two types of these drugs: NNRTIs and NRTIs. Usually, NNRTIs bind to the reverse transcriptase enzyme and prevent the HIV RNA from converting to DNA, thereby preventing it from being copied into the cell's DNA, while the NRTIs incorporate into the viral DNA and prevent it from producing copies of the virus. NRTIs interrupt the HIV replication cycle via competitive inhibition of HIV reverse transcriptase and termination of the DNA chain. ${ }^{8}$ Reverse transcriptase is an HIV-specific DNA polymerase that allows HIV RNA to be transcribed into single-strand and ultimately double-strand proviral DNA and incorporated into the host-cell genome. It is known that proviral DNA chain elongation is necessary before genome incorporation can occur and is accomplished by the addition of purine and pyrimidine nucleosides to the $3^{\prime}$ end of the growing chain. NRTIs are structurally similar to the DNA nucleoside bases and become incorporated into the proviral DNA chain, which results in the termination of proviral DNA formation. ${ }^{9}$ The PIs operate later in the life cycle of HIV and are designed to stop the protease enzyme 
from assembling the new HIV material to be released to infect other cells. ${ }^{9}$

\section{Effects of ART on quality of life of people living with HIV and AIDS}

The use of ART has become the cornerstone of the clinical intervention that is available to prevent transmission and slow progression of HIV infection in individuals living with HIV/AIDS. Interestingly, efforts have begun for a significant scaling up of the use of ART in developing countries, such as those in sub-Saharan Africa, where the epidemic has had its most devastating impact. However, questions have been raised about the use of ART and how it affects the quality of life (QOL) of people living with HIV and AIDS, either negatively or positively. In this review, I examined the effects of ART on the QOL of people living with HIV and AIDS, and how ART has improved the QOL of HIV/AIDS patients.

QOL refers to the degree of excellence in a person's life at any given period that contributes to satisfaction and happiness of the person and benefits society. QOL is multifaceted, incorporating physical, material, psychological, social, and spiritual well-being. ${ }^{11,12}$ It is also important to note that QOL relates both to adequacy of material circumstances and feeling about these circumstances. It is said to include overall feelings of well-being that are closely related to moral happiness and satisfaction. ${ }^{10}$ In addition, as health is generally seen as one of the most important determinants of overall QOL, it has been suggested that QOL may be uniquely affected by specific disease such as HIV/AIDS. ${ }^{11}$

Reports have shown that HIV/AIDS patients face various psychological problems, such as stigma, poverty, depression, substance abuse, and cultural beliefs, which can affect their QOL not only from the view of physical health but also from that of mental and social health, which can cause problems that affect important activities and interests of the persons. ${ }^{12}$ In a study that examined coping, social life, and QOL among HIV/AIDS patients, it was observed that income, emotional, social support, and problem- and perception-oriented coping were related to QOL. ${ }^{13}$ Another study conducted by Swindells et $\mathrm{al}^{14}$ on the relationship between HIV infection and the QOL of HIV-infected persons showed that QOL was influenced by satisfaction with social support and coping style. The development of ART has shifted the perception of HIV/AIDS from a fatal to a chronic and potentially manageable disease. ART is capable of improving survival, reducing the occurrence of HIV-related opportunistic infections, and improving patients' QOL. ${ }^{15}$ Clinical improvement of HIV-infected patients under ART has often been measured by reduction in mortality, opportunistic infection rates, or severe AIDS-related symptoms. ${ }^{16}$ However, overall assessments of QOL among people living with HIV/AIDS have also become a major focus of interest as more efficacious and simpler regimen treatments have become available. Scientifically and clinically, ART has been shown to be highly effective and has the ability to effect significant benefits, which in spite of some negative effects have a positive global impact on the QOL and general health of people living with HIV and AIDS. ${ }^{17}$ According to Mannheimer et al, ${ }^{18}$ significant improvements in mean QOL were seen in HIV patients enrolled at two multicenter antiretroviral clinical trials after 1 and 4 months on new ART regimens, and the improvements persisted for 12 months. In a study by Campos et al, ${ }^{19}$ by using a single facet of the WHOQOLBREF instrument, they were able to assess patients' QOL for 4 months after initiating ART based on the individuals' perceptions, values, and preferences, and they found that a high proportion of the patients reported good or very good QOL after about 4 months of ART (66.4\%) and noticed a significant difference when compared to the baseline values before initiating ART treatment. Overall self-perception of QOL has been shown to be a useful screening item for assessing global QOL, and a lower proportion of good and very good QOL has been observed in HIV/AIDS patients who are not seeking primary health services, compared to individuals who have sought primary health services. ${ }^{20,21,22}$ Previous studies have suggested that a better perception of QOL at baseline is a strong predictor of better QOL after initiating ART, which may relate to a potential carryover effect during the course of treatment. ${ }^{23,24}$ Also, patients with worse QOL at baseline could have higher proportions of anxiety and depression symptoms as well as worse clinical condition for AIDS, and this could negatively affect QOL at follow-up, independent of adherence to ART.

The impact of psychiatric symptoms on the QOL of patients with HIV/AIDS has been reported. ${ }^{25,26}$ Chan et $\mathrm{al}^{27}$ described a significant reduction in psychiatric symptoms when comparing different treatment groups in a representative sample of HIV-infected patients receiving care in the US, including patients receiving ART at baseline, follow-up patients, patients who initiated ART at baseline, patients who were on ART only at baseline, and patients who were not on ART, and showed that ART potentially improves QOL and contributes to enhanced treatment via better adherence to ART. Treatment-related factors were identified to be barriers to a good QOL, and this is consistent with literature that indicates that maintaining the same ART regimen during 
the follow-up period and shows a better QOL than using different ART. ${ }^{22,28}$

Adherence is a critical component for therapeutic success in HIV infection, while improved QOL has been recognized as an important outcome from treatment of HIV; however, adherence to ART has been shown to be the main determinant of biological outcome measures in HIV, including HIV RNA level, CD4 lymphocyte count and genotypic resistance..$^{29,30,31,32}$ Adherence has also been found to predict clinical outcome measures in HIV/AIDS patients as well as mortality, AIDS progression, and hospitalization. ${ }^{33,34}$ The association between QOL and adherence has not been well studied, but ART adherence is known to contribute to the QOL of people living with HIV and AIDS, and QOL is in turn believed to positively influence adherence, as persons with better QOL may have a greater ability to adhere to their ART regimens; studies have also shown that adherence and QOL share some determinants. Both QOL and adherence have been shown to be associated with HIV RNA levels, HIV disease stage, and symptoms. QOL and ART adherence share an inverse relationship with HIV RNA levels: lower adherence rates predict higher HIV RNA levels, and this virological failure has been associated with lower QOL scores. ${ }^{30,35,36}$ According to Mannheimer et al, ${ }^{18}$ participants who reported $100 \%$ ART adherence achieved significantly higher QOL scores at 12 months of follow-up when compared to those with poorer ART adherence, and QOL improved with ART treatment and ART adherence. ${ }^{37}$ In the same study, it was demonstrated that QOL improved over time in $1000 \mathrm{HIV}$-infected persons receiving ART and the improvement was sustained over the 12-month period of ART treatment, but that the improvement was most significant among participants with the highest adherence levels. In a cross-sectional analysis of their results at 4, 8, and 12 months after initiation of ART regimens, significant differences were seen at 4 months for the mental component of the QOL score and at 12 months for both the mental and physical components and in seven of the QOL domains. In each case, those who reported $100 \%$ adherence had the greatest gains, those with $80 \%-99 \%$ adherence levels had smaller benefits, and those with $<80 \%$ adherence had lower QOL scores than at baseline. In this same study, male gender was also found to be associated with an improved physical component of QOL. However, the authors reported that this was not related to differences in access to ART treatment, as all patients received ART treatment. The underlying cause for this gender difference remains unclear and may require further study.

Education, an indicator of socioeconomic status, has also been shown to influence QOL of people living with HIV and
AIDS. Reports have shown that patients with higher education reported better QOL, possibly due to better knowledge about their treatment and disease, access to health services, or functional status. ${ }^{38,39}$ In this author's opinion, this suggests that ART works in combination with such other factors as education and socioeconomic status to effect the desired improvement in the QOL of people living with HIV and AIDS.

In a South African study conducted by Wouters et al, ${ }^{40}$ among 268 patients enrolled in South Africa's public sector ART program for 12 months, it was reported that the physical and emotional QOL of the respondents was high and that the improved QOL observed was sustained over the 12-month study period. The study also evaluated the impact of adverse effects of medication on the patients' physical and emotional QOL, and reported that although adverse effects to ART significantly and negatively influenced patients' physical and emotional QOL, their longitudinal data showed that patients reported significantly fewer adverse effects at follow-up than at baseline. The most cited adverse effects (dizziness, nausea, and skin problems) were related to mild toxicity, which does not require the discontinuation of ART, and adverse effects decrease over time.

Improvement in the QOL domains of cognitive function, physical health, social activities, pain, sleep, feelings, and emotions following ART treatment has been reported..$^{41}$ It has been demonstrated that patients with higher CD4 counts had better QOL, especially with regard to physical health. ${ }^{42,43,44}$ $\mathrm{Wu}$ et $\mathrm{a}^{45}$ and Ware et $\mathrm{a}^{46}$ also reported similar findings, whereas Paton et $\mathrm{al}^{47}$ found that the differences between the disease stages and correlations with CD4 counts extended to nearly all the subscales of physical and mental health. However, some investigators have reported absence of a clear association between scores on psychological domains and the stages of HIV infection, thus signifying the need to combine ART with psychological intervention. ${ }^{48,49}$

Studies from Europe and North America indicate that HIV-infected women frequently become pregnant, and most HIV-infected individuals have fertility desires that change over time..$^{50,51,52}$ A study in Africa indicated that HIV might modify desires but does not eliminate the desire to have children, and that ART use may be associated with increased fertility desires among HIV-infected women, possibly through increased hopes and planning for the future..$^{52}$ The general consensus is that HIV care and ART treatment do encourage HIV-infected women to fall pregnant, because of the belief that it would reduce the risk of vertical transmission of HIV, and to avoid the use of potentially teratogenic drugs, thus ensuring optimal outcomes in these women and 
their children. A study by Myer et $\mathrm{al}^{53}$ showed a significant association between the use of ART and increased incidence of pregnancy. The authors noted that within 4 years of followup, one-third of women who initiated ART experienced a pregnancy, highlighting the fact that ART could improve the QOL of people living with HIV and AIDS, including $\mathrm{HIV}$-infected women who wish to have children. ${ }^{53} \mathrm{~A}$ series of biological and behavioral factors may influence the association between the use of ART and increased incidence of pregnancy. It is possible that the rapid improvements in health and QOL that take place with ART initiation led to increased sexual activity, especially for those with stable partners. ${ }^{54,55}$ Improving health with ART use may contribute to increased fertility desires through psychological mechanisms of increased hopefulness about the future and improved mental health, as well as through increase in sexual activity and new partner acquisition. ${ }^{54}$ It is also possible that improvements in immunological functioning with ART increase female fecundity compared to pre-ART levels.

A study conducted by Kabue et $\mathrm{al}^{56}$ showed that ART improves the growth and survival of HIV-infected individuals. To confirm this, the authors designed a retrospective cohort study to assess clinical factors associated with growth in HIVinfected children on ART in Uganda between July 2003 and March 2006. Height and weight measurements taken pre- and post-ART initiation for at least 6 months were age- and genderstandardized according to Centers for Disease Control and Prevention criteria, and medical records of 749 children receiving ART were analyzed. Descriptive and logistic regression analyses were conducted to identify covariates associated with the risk of either stunting or being underweight. Longitudinal regression analysis with a mixed model using autoregressive covariance structure was used to compare change in height and weight before and after initiation of ART. The mean age of the study population at first visit was reported as 7.5 years, while mean height-for-age, weight-for-age, and weight-for-height percentiles at first visit were 8.6, 7.7, and 7.9, respectively. At last visit, mean height-for-age, weight-for-age, and weightfor-height percentiles were 8.6, 13.3, and 13.8, respectively. A baseline weight-for-age Z-score of 1 or more was protective against stunting (odds ratio [OR] 0.25, confidence interval [CI] 0.18-0.35), while a baseline height-for-age Z-score of 1 or more was protective against becoming underweight (OR 0.75, CI 0.63-0.88). Children in WHO stages II, III, and IV at baseline were 1.5 times more likely to become underweight (OR 1.51, CI 1.07-2.14). The authors noted that initiation of ART resulted in improvement in mean standardized weightfor-age $\mathrm{Z}$-score and weight-for-age percentile $(P<0.001)$, and also that weight-for-age percentile and Z-score improved significantly after initiation of ART. They concluded that the studied pediatric population gained weight more rapidly than height after initiation of ART.

There is both clinical and experimental evidence for direct or indirect negative effects of ARV agents. Some PIs have been observed to reversibly inhibit glucose uptake into muscle and adipose tissue cells at the level of the glucose transport molecule GLUT4, and PIs may also affect hepatic very-low-density lipoprotein secretion through an inhibition of intracellular apoprotein B degradation. ${ }^{56,57}$

NRTIs, especially thymidine analogs, may also have direct toxicity. For instance, fialuridine, the first agent tested in chronic hepatitis B infection was the precedent for NRTI toxicity. Although the drug was effective, it led to a high incidence of subacute liver failure with symptomatic lactic acidosis, and the toxic effect was found to be mitochondrial toxicity relating to inhibition of the effect of DNA polymerase gamma, the DNA polymerase involved in mitochondrial function. ${ }^{58}$ A single-nucleotide polymorphism in the resistin gene has been shown to be associated with a cluster of metabolic alterations, including insulin resistance, dyslipidemia, and lipoatrophy in patients receiving ART. ${ }^{59}$ The identification of lipodystrophy has had significant implications on the management of HIV, and its recognition as a side effect of ART has led to reevaluation of the appropriate time to start ART, delay initiation of ART, and also to different modifications in ART for HIV treatment. Several QOL studies in HIV lipodystrophy have generally shown that patients enjoy a good QOL, including good physical functioning, whereas common complaints that manifested as anxiety and depression were mostly related to the stigma associated with morphological changes. ${ }^{60,61}$ HIV-positive persons may refuse ART or reduce treatment compliance as a result of or in order to avoid the side effects, and as a result, they may experience rebound in viral load or the development of viral resistance, which could consequently lead to disease progression and even to an increased risk of disease transmission, probably by a drug-resistant viral strain. The changes associated with lipodystrophy are associated with cardiovascular disease, and some studies have implicated an increased risk for developing symptomatic cardiovascular disease. ${ }^{62,63}$ Previous studies have suggested that lipodystrophy syndrome in HIV-positive persons on ART is characterized by subcutaneous fat wasting, visceral fat accumulation, lipid abnormalities, and insulin resistance or glucose intolerance, and all of these affect the QOL of people living with HIV and AIDS. ${ }^{64}$ An important observation was 
made by researchers in the Multicenter AIDS Cohort Study, who had the privilege of following 50 persons from a period prior to HIV seroconversion through initiation and follow-up of ART. ${ }^{66} \mathrm{~A}$ fall in total, high-density-lipoprotein cholesterol and low-density-lipoprotein cholesterol concentrations was reported after seroconversion and persisted as immune function declined and initiation of ART led to increase in total and low-density-lipoprotein cholesterol.

The effects of ART on oxidative stress have been investigated. Ngondi and colleagues reported that combined ART increased the oxidative stress in HIV and AIDS patients receiving ART. ${ }^{67}$ ART may increase oxidative stress levels above levels caused by the HIV itself. In HIV infection, oxidative stress may enhance viral replication by activating nuclear transcription factors, which leads to viral gene expression. In HIV-infected adults, zidovudine was shown to promote oxidative damage to DNA. ${ }^{67}$ It is been noted that ART may induce an increase in oxidant generation, decrease in antioxidant protection, and a failure to repair oxidative damage. Ngondi et $\mathrm{al}^{66}$ also noticed that increased oxidative stress brought about by HIV as well as the use of ART was paralleled by significant decreases in glutathione level, albumin, and vitamin C. It should be noted that abnormally high levels of free radicals as well as a simultaneous reduction in antioxidant defense mechanisms can lead to damage of cellular organelles and enzymes and increased lipid peroxidation. Under normal conditions, several cellular antioxidant systems exist to defend against oxidative stress and maintain the redox balance of the cell. However, ART may reduce glutathione synthesis, enhance glutathione utilization, or limit intracellular reduction of its reduced form and as a consequence of glutathione deficiency, a number of related functions may be impaired, such as a decrease in reducing capacity, protein biosynthesis, immune function, accumulation of lipid peroxidation products, and detoxification capacity. ${ }^{68-70} \mathrm{~A}$ reduced detoxification capacity in the liver may lead to accumulation of hepatotoxic metabolites, leading to liver damage and thus reduction in the QOL of people living with HIV and AIDS. ${ }^{68,69}$ Weight loss and wasting may still be observed in HIV patients on ART. One study reported that $10 \%$ of ART patients were underweight with chronic diarrhea. ${ }^{66}$

\section{Conclusion and recommendation}

The evolution of ART treatment of HIV/AIDS as a chronic infection/disease presents challenges for patients and health-care professionals, and measures of QOL can provide important information in behavioral and clinical studies of ART. The relationships between ART and multidimensional construct of the QOL of people living with HIV and AIDS is complex; however, this review provides a framework for practical and theoretical understanding of the relationships, thus contributing to the understanding of the impact of ART on the various aspects of the QOL of people living with HIV and AIDS. Focus should be on how to maximize the positive effects of ART, especially in resource-poor settings, and particularly in developing countries. There is a need to provide more support to HIV/AIDS patients living in rural areas and to organize awareness programs that can address the issue of stigma and discrimination, since such awareness programs could contribute to a better QOL in HIV/AIDS patients. Clinical assessment of adverse reactions during the course of ART treatment and careful monitoring after any ART switch could contribute to a better QOL, improve the patientdoctor relationship, and potentially maintain adherence with fewer undesired side effects.

\section{Disclosure}

The author reports no conflicts of interest in this work.

\section{References}

1. Joint United Nations Programme on HIV/AIDS (UNAIDS). Report on the Global AIDS Epidemic. Geneva: UNAIDS; 2008.

2. Cadman J, Arboleda C. Once or Twice: A Guide to Medication Dosing for HIV Infection. Washington: National Minority AIDS Council; 2001.

3. Shen L, Peterson S, Sedaghat A, et al. Dose-response curve slope sets class-specific limits on inhibitory potential of anti-HIV drugs. Nat Med. 2008; 14:762-766.

4. Weller IV, Williams IG. ABC of AIDS: antiretroviral drugs. BMJ. 2001;322:1410-1412.

5. Clavel F, Hance AJ. HIV drug resistance. $N$ Engl J Med. 2004;350:1023-1035.

6. Cooper RD, Wiebe N, Smith N, Keiser P, Naicker S, Tonelli M. Systematic review and meta-analysis: renal safety of tenofovir disoproxil fumarate in HIV-infected patients. Clin Infect Dis. 2010;51:496-505

7. Panel on Antiretroviral Guidelines for Adults and Adolescents. Guidelines for the Use of Antiretroviral Agents in HIV-1-Infected Adults and Adolescents. Washington: Department of Health and Human Services; 2011.

8. Cox SW, Apéria K, Albert J, Wahren B. Comparison of the sensitivities of primary isolates of HIV type 2 and HIV type 1 to antiviral drugs and drug combinations. AIDS Res Hum Retroviruses. 1994; 10:1725-1729.

9. Elion RA, Witt MD. Nucleoside and nucleotide reverse transcriptase inhibitors in the treatment of HIV: focus on efficacy. 2003. Available from: http://www.medscape.org/viewarticle/465383_1. Accessed May 18, 2012.

10. Newell M. A Guide to Rating Scales and Questionnaires. New York: Oxford University Press; 1987.

11. Fanning M. Validation of a Quality of Life Instrument for Patients with HIV Infection. Ottawa: Health and Welfare Canada; 1994.

12. Aranda-Naranjo B. Quality of life in HIV-positive patients. $J$ Assoc Nurses AIDS Care. 2004;15:20-27. 
13. Friedland J, Rewick R, McColl M. Coping and social support as determinants of quality of life in HIV/AIDS. AIDS Care. 1996;8:15-31.

14. Swindells S, Mohr J, Justis JC, Berman S, Squier C, Wagener MM. Quality of life in patients with human immunodeficiency virus infection: impact of social support, coping style and hopelessness. Int JSTD AIDS. 1999;10:383-391.

15. Marins JRP, Jamal LF, Chen SY, et al. Dramatic improvement in survival among adult Brazilian AIDS patients. AIDS. 2003; 17:1675-1682.

16. Crum NF, Riffenburgh RH, Wegner S, et al. Comparisons of causes of death and mortality rates among HIV-infected persons: analysis of the pre, early and late HAART eras. J Acquir Immune Defic Syndr. 2006;41:194-200.

17. Burgoyne RW, Tan DH. Prolongation and quality of life for HIVinfected adults treated with HAART: a balancing act. J Animicrob Chemother. 2008;61:469-473.

18. Mannheimer SB, Matts J, Telzak E, et al. Quality of life in HIV-infected individuals receiving antiretroviral therapy is related to adherence. AIDS. 2005; 17:10-22.

19. Campos LN, César CC, Guimarães MDC. Quality of life among HIVinfected patients in Brazil after initiation of treatment. Clinics (Sao Paulo). 2009;64:867-875.

20. Carr AJ, Thompson PW, Kirwan JR. Quality of life measures. Br J Rheumatol. 1996;35:275-281.

21. Crane HM, Van Rompaey SE, Dillingham PW, Herman E, Diehr P, Kitahata MM. A single-item measure of health-related quality of life for HIV-infected patients in routine clinical care. AIDS Patient Care STDS. 2000;20:161-174.

22. Fleck MPA, Lima AFB, Louzada S, et al. Association of depressive symptoms and social functioning in primary care service, Brazil. Rev Saude Publica. 2002;36:431-438.

23. Carrieri P, Spire B, Duran S, et al. Health-related quality of life after 1 year of highly active antiretroviral therapy. J Acquir Immune Defic Syndr. 2003;32:38-47.

24. Jia H, Uphold CR, Wu S, Chen GJ, Duncan PW. Predictors of changes in health-related quality of life among men with HIV infection in the HAART era. AIDS Patient Care STDS 2005;19:395-405.

25. Kemppainem JK. Predictors of quality of life in AIDS patients. JAssoc Nurses AIDS Care. 2001;12:61-70.

26. Tostes MA, Chalub M, Botega NJ. The quality of life of HIVinfected women is associated with psychiatric morbidity. AIDS Care. 2004; 16:177-186.

27. Chan KS, Orlando M, Joyce G, et al. Combination antiretroviral therapy and improvements in mental health: results from a nationally representative sample of persons undergoing care for HIV in the United States. J Acquir Immune Defic Syndr. 2003;33:104-111.

28. Preau M, Leport C, Salmon-Ceron D, et al. Health-related quality of life and patient-provider relationships in HIV-infected patients during the first three years after starting PI-containing antiretroviral treatment. AIDS Care. 2004;16:649-661.

29. Wu AW. Quality of life assessment comes of age in the era of HAART. AIDS. 2000;14:1449-1451.

30. Gifford AL, Bormann JE, Shively MJ, Wright BC, Richman DD, Bozzette SA. Predictors of self-reported adherence and plasma HIV concentrations in patients on multidrug antiretroviral regimens. J Acquir Immune Defic Syndr. 2000;23:386-395.

31. Paterson DL, Swindells S, Mohr J, et al. Adherence to protease inhibitor therapy and outcomes in patients with HIV infection. Ann Int Med. 2000;133:21-30

32. Raboud JM, Harris M, Rae S, Montaner JS. Impact of adherence on duration of virological suppression among patients receiving combination antiretroviral therapy. HIV Med. 2002;3:118-124.

33. Hogg RS, Yip B, Chan KJ, et al. Rates of disease progression by baseline CD4 cell count and viral load after initiating triple-drug therapy. JAMA. 2001;286:2568-2577.
34. Morgan D, Mahe C, Mayanja B, Okongo JM, Lubega R, Whitworth JAG. HIV-1 infection in rural Africa: is there a difference in median time to AIDS and survival compared with that in industrialized countries? AIDS. 2002;16:597-603.

35. Mannheimer S, Friedland G, Matts J, Child C, Chesney M. The consistency of adherence to antiretroviral therapy predicts biologic outcomes for human immunodeficiency virus-infected persons in clinical trials. Clin Infect Dis. 2002;34:1115-1121.

36. Nieuwkerk PT, Reijers MH, Weight HM, Lange JM, Sprangiers MA. Quality of life in maintenance versus prolonged induction for HIV. JAMA. 2000;284:178-179.

37. Fumaz CR, Tuldra A, Ferrer MJ, et al. Quality of life, emotional status and adherence of HIV-1-infected patients treated with efavirenz versus protease inhibitor-containing regimens. J Acquir Defic Syndr. 2002;29:244-253.

38. Prieto-Flores ME, Fernández-Mayoralas G, Rojo-Pérez F, et al. Effects of sociodemographic factors on emotional and psychological status of HIV/AIDS patients on ART. Rev Esp Salud Publica. 2008;82:301-313.

39. Liu C, Johnson L, Ostrow D, Silvestre A, Visscher B, Jacobson LP Predictors for lower quality of life in the HAART era among HIVinfected men. J Acquir Immune Defic Syndr. 2006;42:470-477.

40. Wouters E, Heunis C, Van rensburg D, Meulemans H. Physical and emotional health outcomes after 12 months of public-sector antiretroviral treatment in the Free State Province of South Africa: a longitudinal study using structural equation modelling. BMC Public Health. 2009;9:103.

41. Kohli RM, Sane S, Kumar K, Paranjape RS, Mehendale SM. Modification of medical outcome study (MOS) instrument for QOL assessment and its validation in HIV infected individuals in India. Indian J Med Res. 2005;122:297-304.

42. Badia X, Podzamczer D, Garcia M, López-Lavid C C, Consiglio E. A randomized study comparing instruments for measuring health related quality of life in HIV-infected patients. AIDS. 2001;13:1727-1735.

43. Gill CJ, Griffith JL, Jacobson D, Skinner S, Gorbach SL, Wilson IB. Relationship of HIV viral loads, CD4 counts and HAART use to health related quality of life. J Acquir Immune Defic Syndr. 2002;30:485-492.

44. Campsmith MI, Nakashima AK, Davidson AJ. Self reported health related quality of life in persons with HIV infection:results from multisites interview project. Health Qual Life Outcomes. 2003;1:12.

45. Wu, AW, Rubin HR, Mathews WC, et al. A health status questionnaire using 30 items from the medical outcomes study. Preliminary validation in persons with early HIV infection. Med Care. 1991;29:786-798.

46. Ware JE, Kosinski M, Gandek B. The factor structure of SF-36 health survey in 10 countries: results from the IQOLA project. International Quality of Life Assessment. J Clin Epidemiol. 1997;51:1159-1165.

47. Paton NI, Chapman CA, Chan SP, et al. Validation of the Medical Outcome Study HIV Health Survey as a measure of quality of life in HIVinfected patients in Singapore. Int J STD AIDS. 2002;13:456-461.

48. Smith MY, Feldman J, Kelly P, DeHovitz JA, Chirgwin K, Minkoff H. Health-related quality of life of HIV-infected women: evidence for the reliability, validity and responsiveness of the Medical Outcomes Study Short-Form 20. Qual Life Res. 1996;5:47-55.

49. Wu AW, Revicki DA, Jacobson D, Malitz FE. Evidence for reliability, validity and usefulness of the Medical Outcomes Study HIV Health Survey (MOS-HIV). Qual Life Res. 1997;6:481-493.

50. Massad LS, Springer G, Jacobson L, et al. Pregnancy rates and predictors of conception, miscarriage and abortion in US women with HIV. AIDS. 2004;18:281-286.

51. Fiore S, Heard I, Thorne G, Savasi V, Coll RL, Miu A. Fertility desires and intention of HIV-positive women living in Europe. Hum Reprod. 2001;23:2140-2144.

52. Bah'him YM, Oguntibeju OO, Lewis HA, Mokwena K. Factors associated with pregnancies among HIV-positive women on prevention of mother-to-child transmission programme. West Indian Med J. 2010;59:362-368. 
53. Myer L, Carter RJ, Katyal M, et al. Impact of antiretroviral therapy on incidence of pregnancy among HIV-infected women in Sub-Saharan Africa: a cohort study. PLoS Med. 2010;7:137.

54. Kaida A, Andia I, Maier M, et al. The potential impact of antiretroviral therapy on fertility in sub-Saharan Africa. Curr HIV/AIDS Rep. 2006;3:187-194.

55. Kaida A, Gray G, Bastos FI, et al. The relationship between HAART use and sexual activity among HIV-positive women of reproductive age in Brazil, South Africa and Uganda. AIDS Care. 2008;20:21-25.

56. Kabue MM, Kekitiinwa A, Maganda A, Risser JM, Chan W, Kline MW. Growth in HIV-infected receiving antiretroviral therapy at a paediatric infectious diseases clinic in Uganda. AIDS Patient Care STDS. 2008;22:245-251.

57. Murata H, Hruz PW, Muecker M. The mechanism of insulin resistance caused by HIV protease inhibitor therapy. J Biol Chem. 2000;275:20251-20254.

58. Liang JS, Dister O, Cooper DA, et al. HIV protease inhibitors protect apolipoprotein B from degradation by the proteosome: a potential mechanism for protease inhibitor-induced hyperlipidaemia. Nat Med. 2001;7:1327-1331.

59. Blumer RM, van Vonderen MG, Sutinen J, et al. Zidovudine-lamivudine contributes to insulin resistance within 3 months of starting combination antiretroviral therapy. AIDS. 2008;22:227-236.

60. Ranade K, Geese WJ, Noor M, et al. Genetic analysis implicates resistin in HIV lipodystrophy. AIDS. 2008;22:1561-1568.

61. Marin A, Casado JL, Aranzabal L, et al. Validation of a specific questionnaire on psychological and social repercussions of the lipodystrophy syndrome in HIV-infected patients. Qual Life Res. 2006;15:767-775.
62. Ammassari A, Murri R, Pezzotti, et al. Self-reported symptoms and medication side effects influence adherence to highly active antiretroviral therapy in persons with HIV infection. J Acquir Immune Defic Syndr. 2001;28:445-449.

63. Friis-Møller N, Sabin CA, Weber R, et al. Combination antiretroviral therapy and the risk of myocardial infarction. $N$ Engl J Med. 2003;349:1993-2003.

64. Klein D, Hurley LB, Quesenberry CP Jr, Sidney S. Do protease inhibitor increase the risk for coronary heart disease in patients with HIV-1 infection? J Acquir Immune Defic Syndr. 2002;30:471-477.

65. Carr A, Emery S, Law M, et al. An objective case definition of lipodystrophy in HIV-infected adults: a case-control study. Lancet. 2003;361:726-735.

66. Riddler SA, Smit E, Cole SR, et al. Impact of HIV infections and HAART on serum lipids in men. JAMA. 2003;289:2978-2982.

67. Ngondi JL, Oben J, Forkah DM, Etame LU, Mbanya D. The effect of different combination therapies on oxidative stress markers in HIV-infected patients in Cameroon. AIDS Res Ther. 2006;3:1-7.

68. Allard JP, Aghdassi E, Chau J, et al. Effects of vitamin E and C supplementation on oxidative stress and viral load in HIV-infected subjects. AIDS. 1998;12:1653-1659.

69. Sen SK. Cellular thiols and redox-regulated signal transduction. Curr Top Cell Regul. 2000;36:1-30.

70. Hayes JD, McLellan LI. Glutathione and glutathione-dependent enzymes represent a co-ordinately regulated defence against oxidative stress. Free Radic Res. 1999;31:273-300.
HIV/AIDS - Research and Palliative Care

\section{Publish your work in this journal}

HIV/AIDS - Research and Palliative Care is an international, peerreviewed open-access journal focusing on advances in research in HIV, its clinical progression and management options including antiviral treatment, palliative care and public healthcare policies to control viral spread. The journal welcomes original research, basic science,

\section{Dovepress}

clinical \& epidemiological studies, reviews \& evaluations, expert opinion \& commentary, case reports \& extended reports. The manuscript management system is completely online and includes a very quick and fair peer-review system. Visit http://www.dovepress.com/ testimonials.php to read real quotes from published authors. 